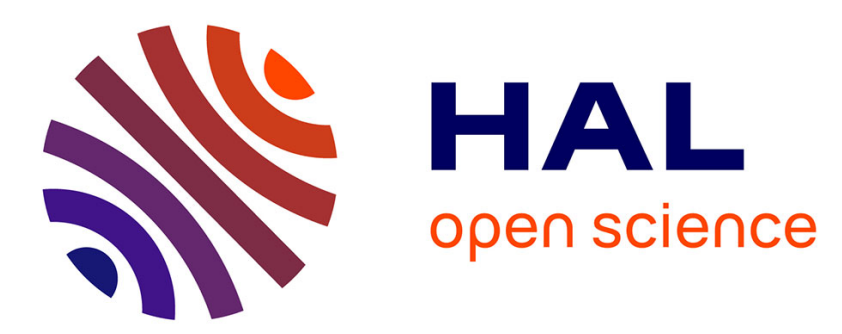

\title{
Higher-Order Continuum Theory Applied to Fracture Simulation of Nano-scale Intergranular Glassy Film
}

Yang Yang, W.Y. Ching, Anil Misra

\section{To cite this version:}

Yang Yang, W.Y. Ching, Anil Misra. Higher-Order Continuum Theory Applied to Fracture Simulation of Nano-scale Intergranular Glassy Film. 2011. hal-00556184

\section{HAL Id: hal-00556184 \\ https://hal.science/hal-00556184}

Preprint submitted on 15 Jan 2011

HAL is a multi-disciplinary open access archive for the deposit and dissemination of scientific research documents, whether they are published or not. The documents may come from teaching and research institutions in France or abroad, or from public or private research centers.
L'archive ouverte pluridisciplinaire HAL, est destinée au dépôt et à la diffusion de documents scientifiques de niveau recherche, publiés ou non, émanant des établissements d'enseignement et de recherche français ou étrangers, des laboratoires publics ou privés. 


\title{
Higher-Order Continuum Theory Applied to Fracture Simulation of Nano-scale Intergranular Glassy Film
}

\author{
Yang Yang ${ }^{1}$, W. Y. Ching ${ }^{2}$ and Anil Misra ${ }^{3 *}$, M.ASCE \\ ${ }^{1}$ Ph.D. student, Faculty of Engineering, China University of Geosciences, Wuhan, 430074, China, and Visiting \\ Scholar, Department of Civil, Environmental and Architectural Engineering, the University of Kansas, 1530 W. \\ 15th Street, Lawrence, KS, USA. Email: yangyang@ku.edu \\ ${ }^{2}$ Curators' Professor Department of Physics, University of Missouri-Kansas City, MO 64110, USA, Email: \\ ChingW@umkc.edu. \\ $3^{3 *}$ Corresponding Author, Professor, Department of Civil, Environmental and Architectural Engineering, The \\ University of Kansas, 1530 W. 15th Street, Lawrence, KS 66045, USA., Ph: (785) 864-1750, Fax: (785) \\ 864-5631, Email: amisra@ku.edu
}

\begin{abstract}
Complex grain-boundary structures, such as the 1-2 nm thick intergranular glassy films (IGF), play a prominent role in the failure behavior of nano-phased ceramics. The IGF plays the role of an imperfection and serves as the location of strain localization and failure. We have recently performed "theoretical" mechanical loading experiments on very large atomic models of IGF in silicon nitride using $a b$ initio simulation to obtain their failure behavior. The $a b$ initio simulations yield characteristic post-peak softening accompanied by strain localization zone. In this paper a micro-structural granular mechanics based higher-order continuum theory is applied to model the failure behavior of these types of material systems. The results obtained from the $a b$ initio simulations are compared with those predicted by the higher order continuum theory.
\end{abstract}

Keywords: Higher-order theory; granular mechanics; $a b$ initio; atomic models; strain softening; element-free Galerkin. 


\section{Introduction}

Nano-phased ceramics are attracting wide attention in structural and electronics applications as a class of materials with extraordinary mechanical properties (Zhu et al. 2009; Koch et al. 2007). By reducing the grain-sizes to nano-scale and manipulating materials at atomic scales, ceramics are being sought that can attain theoretical strengths predicted for single defect-free crystals. However, the understanding of the mechanical properties of these materials at their atomic scales remains insufficient. A unique feature of nano-phased ceramics is the existence of thin glassy films between crystallites with a narrowly distributed width of about 1-2 nm (Luo 2007; Subramaniam et al. 2006). Although these thin intergranular glassy films (IGF) occupy only a very small fraction of the overall composition and volume, they have a profound effect on the physical and mechanical properties of the bulk materials. For example, small amounts of rare earth doping which usually reside at or near the IGF results in significantly higher strength for bulk material (Shibata et al. 2004; Ziegler et al. 2004).

The role that microstructures, such as crystal defects and grain boundaries, play in determining the mechanical behavior of materials has been widely recognized and continue to be researched vigorously (Phillips 2001; Sutton and Balluffi 1995; Koch et al. 2007; Ovid'ko 2007). Among the various microstructures, IGF characterize many nano-phased ceramics irrespective of their synthesis process (Luo 2007; Pan 1996). The mechanics of these larger complex microstructures, such as IGF, have been rarely investigated. In addition, as these new nano-phased ceramics are developed and the devices based upon them miniaturized, there has been an increasing interest in understanding their deformation and failure behavior from the viewpoint of atomic-scale mechanisms (see Huang and Van Swygenhoven 2009 and papers therein). At the fundamental-level, the deformation and failure mechanics of these materials can be traced to the electronic structure and the bonding between atoms. This is particularly true for covalently bonded solids, such as the ceramic materials. The thin size of the IGF and a strong 3-dimensional nature of grain-interactions make it extremely difficult to study their mechanical behavior using high-resolution experimental techniques.

The IGF and its interface with crystals is composed of a variety of defective structures as they are typically formed of under or over-coordinated atoms with bond-length and bond-angle distortions. In many cases, the IGF offer a more favorable failure mechanism in real systems as opposed to crystal defects or crystal grain boundaries. Since the IGF atomic structures are varied and complex, simulations that are based upon fundamental methods can best reveal the true nature of deformation and failure mechanism. We have recently performed $a b$ initio simulation of deformation and failure using very large atomic models of IGF in silicon nitride (Ching et al. 2009, 2010; Misra et al. 2007; Chen et al. 2005). Silicon nitride is among the class of lightweight, hard nano-phased ceramics being actively sought for applications as advanced structural and electronic material. Using the results from these highly accurate $a b$ initio calculations, we have explored deformation and failure of systems that have glassy materials interfacing crystalline structures. These ab initio results show that failure initiates within the IGF or its interface with the crystal and develops into a failure band of finite thickness. The failure is accompanied by strain softening similar to that observed at macro-scales for brittle materials. 
$A b$ initio solutions for such complex structures cannot be obtained routinely even with the currently available unprecedented computational resources and such simulations are only possible for relatively small atomic systems. Clearly, such ab initio simulations of macro (meso)-scale mechanical behavior are not feasible for nano-phased materials that are suffused with IGF type structures. Appropriate continuum models are needed that can greatly reduce the computational needs and provide accurate simulations. However, traditional continuum approaches, including gradient theories, suffer from a variety of drawbacks including numerical instability, mesh sensitivity, and ambiguous material constants such as the length scale parameter or the higher order moduli. The objective of this paper is to address the deficiencies of the current approaches by developing micro-structural granular mechanics based higher-order continuum theory. In this paper, we present a micro-structural granular mechanics approach for obtaining the constitutive coefficients such that the internal length scale parameter reflects the natural granularity of the underlying microstructure. In contrast to most conventional gradient approaches, the present higher order theory includes strain gradients and their conjugate higher-order stress. We derive the required constitutive relationships, the governing equations and its weak form for this higher-order theory. An Element-free Galerkin (EFG) formulation is then applied for the discretization of the system governing equations. The derived method is applied to simulate the fracture process of the IGF model and the results are compared to those obtained from ab initio simulations.

\section{Higher-order stress/strain model}

There is a wide recognition that numerical solutions of materials that exhibit strain softening pose significant challenges. For example, finite-element solutions suffer from numerical instabilities and severe mesh sensitivity (see among others Pietruszczak and Mroz 1981; Bažant et al. 1984; Sandler 1984; Frantziskonis and Desai 1987; de Borst et al. 1993; Chen et al. 2000) and lead to physically unrealistic results with increasing mesh refinement (Bažant 1976; Nemes and Spéciel 1996). A number of approaches have been proposed to address these numerical challenges. These include the various forms of micropolar, non-local, viscoplastic and gradient theories (Mindlin 1969; Chang and Ma 1990; Fleck and Hutchinson 1993, Steinmann 1994; Sandler 1984; Wu and Freund 1984; Needleman 1988; Sluys and de Borst 1992; Nemes and Spéciel 1996; Bažant et al. 1984; Belytschko et al. 1986; Bažant and Pijaudier-Cabot 1988; Valanis 1991; Murakami et al. 1993; de Vree et al. 1995; Chen et al. 2000).

Among the various approaches proposed for modeling strain softening behavior, gradient theories have emerged as a viable method. The attractions of the gradient methods are its simplicity as no rotational degree of freedom or time effects are required, nor is there any dependence on unknown 'weak zones' within the solid, and the difficult to determine influence functions for the convolution integrals appearing in the classical non-local models are avoided (Triantafyllidis and Bardenhagen 1993). In addition, this approach follows strict locality in a mathematical sense (Peerlings et al. 1996) and incorporates an inherent characteristic length scale that determines the size of the localization zone. Gradient models have been widely used for modeling softening failure behavior (de Borst and Mühlhaus 1992; Pamin 1994; de Borst et al. 1995; Peerlings et al. 1996; Altan and Aifantis 1997; Chang et al. 2002; Sluys 1992; Sluys et al. 1993; Chang and Gao 1997; Chang et al. 1998; Suiker et al. 2001a, b). 
In contrast to most currently used gradient theories, the approach developed in this paper considers both the higher-order strain and the higher-order stress terms (Germain 1973). This type of model appears to unconditionally maintain the stability and, therefore, offers a more robust approach (Chang et al. 2002). However, this approach has been rarely employed mainly because of its numerical complexity and ambiguous material constants. In this paper, we focus upon the derivation of a first-order theory that includes the usual Cauchy stresses and strains termed as zeroth-order tensors in constitutive model, and the first-order gradient of strain and its conjugated first-order stress. We derive the higher-order constitutive laws on the basis of a microstructural granular mechanics approach (Chang and Gao 1995; Mühlhaus and Oka 1996; Suiker et al. 2001a, b). The macroscopic material model of this pseudo-granular material is obtained in terms of the microscopic material properties. As a result, first-order gradient damage constitutive models are derived such that a so-called internal length scale, i.e. the particle radius, is incorporated directly into the model to reflect the granularity of the underlying microstructure. We then derive the governing equations and their weak form for this first-order gradient theory.

\section{Higher-order constitutive law using microstructural granular mechanics}

A macroscopic continuum is postulated to have a granular microstructure consisting of a set of interacting particles whose centroids represent material points as depicted in Fig. 1. Under an applied load on a sample of such a material, the conceptual grains may undergo translation or rotation. The relative displacement, $\delta_{i}$, between two nearest neighbor particles $\boldsymbol{n}$ and $\boldsymbol{p}$ (Chang and Misra 1990, Misra and Yang 2010) is given by

$$
\delta_{i}=u_{i}^{n}-u_{i}^{p}+e_{i j k}\left(\omega_{j}^{n} r_{k}^{n}-\omega_{j}^{p} r_{k}^{p}\right)
$$

where $u_{i}$ =particle displacement; $\omega_{j}=$ particle rotation; $r_{k}=$ vector joining the centroid of particle to the contact point; superscripts refer to the interacting particles; $e_{i j k}=$ the permutation symbols. Note that all subscripts follow the summation convention of tensor.

The contact force $f_{i}^{c}$ between two particles may be related to the relative displacement $\delta_{j}^{c}$ through the contact stiffness $K_{i j}^{c}$ as

$$
f_{i}^{c}=K_{i j}^{c} \delta_{j}^{c}
$$

with $K_{i j}^{c}$ written in terms of the stiffness components in the normal direction $K_{n}$ and that in the tangential direction $K_{w}$ as

$$
K_{i j}^{c}=K_{n}^{c} n_{i} n_{j}+K_{w}^{c}\left(s_{i} s_{j}+t_{i} t_{j}\right)
$$

where $n, s, t$ are the unit base vectors of the local coordinate system constructed at each contact. Vector $n$ is normal to the contact plane and the other two orthogonal vectors, $s$ and $t$, are on the contact plane which are 
given by

$\left\{\begin{array}{l}n=\cos \gamma e_{1}+\sin \gamma \cos \phi e_{2}+\sin \gamma \sin \phi e_{3} \\ s=\frac{d n}{d \gamma}=-\sin \gamma e_{1}+\cos \gamma \cos \phi e_{2}+\cos \gamma \sin \phi e_{3} \\ t=n \times s=-\sin \phi e_{2}+\cos \phi e_{3}\end{array}\right.$

The strain energy density in a representative volume $V$ of this pseudo-granular material can be written as

$W=\frac{1}{2 V} \sum_{c=1}^{N} f_{i}^{c} \delta_{i}^{c}$

where $N$ refers to the total number of inter-particle contacts. To develop a continuum model for the behavior of a particle assembly, we associate the discrete displacement, $u_{i}^{n}$, of the $n$th particle to the displacement of the centroid, $x_{i}^{n}$, of the $n$th particle, $u_{i}\left(x_{i}{ }^{n}\right)$. Following the approach by Chang and Liao (1990), Taylor series expansions is used for the displacement field. Thus, the displacement at particle $n$ can be estimated using the gradients at a reference point, $x^{0}$, which is defined as the center of the representative volume as follows:

$u_{i}\left(x^{n}\right)=u_{i}\left(x^{0}\right)+u_{i, j}\left(x^{0}\right) x_{j}+\frac{1}{2} u_{i, j k}\left(x^{0}\right) x_{j} x_{k}$

where the derivatives of third- and higher-order are neglected. Ignoring the particle rotations and substituting Eq. (6) into Eq. (1) we get

$\delta_{i}^{c}=u_{i}\left(x^{n}\right)-u_{i}\left(x^{p}\right)=u_{i, j} L_{j}^{c}+u_{i, j k} J_{j k}^{c}$

where the geometric quantities

$L_{j}^{c}=x_{j}^{n}-x_{j}^{p}$

$J_{j k}^{c}=\frac{1}{2}\left(x_{j}^{n} x_{k}^{n}-x_{j}^{p} x_{k}^{p}\right)$

Assuming that the origin of local coordinates is located at the $p$ th particle, and the pseudo-particle radius is uniform denoted by, $r$, Eqs. (8a)-(8b) are reduced to

$$
\begin{aligned}
& L_{j}^{c}=2 r n_{j}^{c} \\
& J_{j k}^{c}=\frac{1}{2} L_{j}^{c} L_{k}^{c}
\end{aligned}
$$

Now considering the zeroth and the first-order strain measure, $\varepsilon$, as

$\varepsilon_{i j}^{0}=u_{i, j} \quad \varepsilon_{i j k}^{I}=u_{i, j k}$

the conjugate stress measures $\sigma$ can be defined as

$$
\sigma_{i j}^{0}=\frac{\partial W}{\partial \varepsilon_{i j}^{0}} \quad \sigma_{i j k}^{I}=\frac{\partial W}{\partial \varepsilon_{i j k}^{I}}
$$

By combining Eqs. (7), (9a)-(11), following set of constitutive equations is obtained: 
$\sigma_{i j}^{0}=C_{i j q m} \varepsilon_{q m}^{o}+B_{i j q m n} \varepsilon_{q m n}^{I}$

$\sigma_{i j k}^{I}=B_{i j k q m} \varepsilon_{q m}^{0}+D_{i j k q m n} \varepsilon_{q m n}^{I}$

which is further simplified as follows for the case of material with central symmetry such that constitutive tensor, $B_{i j q m n}=0$ (Chang et al. 2002):

$\sigma_{i j}^{0}=C_{i j k l} \varepsilon_{k l}^{0}$

$\sigma_{i j q}^{1}=D_{i j g k l m} \varepsilon_{k l m}^{1}$

Using Eqs (5), (7)-(11), the constitutive tensors in Eqs. (13a) and (13b) can be expressed in terms of the fabric measures and the inter-granular stiffness as

$$
\begin{aligned}
& C_{i j q m}=\frac{1}{2 V} \sum_{c=1}^{N} L_{j}^{c} K_{i q}^{c} L_{m}^{c} \\
& D_{i j k q m n}=\frac{1}{8 V} \sum_{c=1}^{N} L_{j}^{c} L_{k}^{c} K_{i q}^{c} L_{m}^{c} L_{n}^{c}
\end{aligned}
$$

Considering the symmetry of the higher-order stress and strain tensors, the fourth-rank and sixth-rank constitutive tensors have to satisfy the following symmetries

$$
\begin{aligned}
& C_{i j k l}=C_{k l i j} ; C_{i j k l}=C_{j i k l}=C_{i j l k} \\
& D_{i j q k l m}=D_{k l m i j q} ; D_{i j g k l m}=D_{j i q k l m}=D_{i j q l k m}
\end{aligned}
$$

Since the representative volume consists of a large number of particles, a summation of any quantity over all particle contacts within the volume can be expressed in an integral form by introducing a directional density function, $\xi(\gamma, \phi)$ (Chang and Misra 1990). For a suitably large representative volume with a large number of contacts, recalling Eq. (9a), the summation in Eqs. (14a) and (14b) may be recast into integral forms as

$$
\begin{aligned}
& C_{i j k l}=\frac{2 r^{2} N}{V} \int_{\Omega} n_{j}^{c} K_{i k}^{c} n_{l}^{c} \xi(\gamma, \phi) d \Omega \\
& D_{i j q k l m}=\frac{2 r^{4} N}{V} \int_{\Omega} n_{j}^{c} n_{q}^{c} K_{i k}^{c} n_{l}^{c} n_{m}^{c} \xi(\gamma, \phi) d \Omega
\end{aligned}
$$

where the integration $\int_{\Omega}(\quad) d \Omega=\int_{0}^{2 \pi} \int_{0}^{\pi}() \sin \gamma d \gamma d \phi$; and $N \xi(\gamma,) \phi d \Omega=$ the number of contacts in the 
interval $\Omega$ to $\Omega+d \Omega$. For isotropic micro-structure

$\xi(\gamma, \phi)=\frac{1}{4 \pi}$

and closed form expressions for the constitutive coefficients can be derived in terms of the Young's modulus, Poisson's ratio and the particle size. Substituting Eqs.(3), (4) and (18) into (17a) and integrating we arrive at the zeroth-order constitutive constants, $C_{i j k l}$, as

$$
\begin{aligned}
& C_{1111}=\frac{a}{15}\left(3 K_{n}+2 K_{w}\right) \\
& C_{1122}=\frac{a}{15}\left(K_{n}-K_{w}\right) \\
& C_{1212}+C_{1221}=\frac{a}{15}\left(K_{n}+\frac{3}{2} K_{w}\right)
\end{aligned}
$$

where $a=2 r^{2} N / V$ represents the density of the packing structure. In addition, the following identities for zeroth-order elastic moduli hold:

$$
\begin{aligned}
& C_{1111}=C_{2222} \\
& C_{1122}=C_{2211} \\
& C_{1212}=C_{2121}
\end{aligned}
$$

The constitutive constants results in the following relations between material properties and components of pseudo-bond stiffness (Chang and Misra 1990; Chang and Gao 1995):

$$
\begin{aligned}
& E=a\left(\frac{K_{n}\left(2 K_{n}+3 K_{w}\right)}{3\left(4 K_{n}+K_{w}\right)}\right) \\
& v=\frac{K_{n}-K_{w}}{4 K_{n}+K_{w}}
\end{aligned}
$$

Eqs.(21) and (22) can be rearranged to give the pseudo-bond stiffness

$$
\begin{aligned}
& K_{n}=\frac{3 E}{a(1-2 v)} \\
& K_{w}=\frac{3 E(1-4 v)}{a(1-2 v)(1+v)}
\end{aligned}
$$

By combing Eqs. (3), (4), (17b), (18), (23) and (24) and using a similar algebra, the components of first-order constitutive constants $D_{i j q k l m}$ can be obtained as

$$
D_{111111}=\frac{3 r^{2} E(7-3 v)}{35(1-2 v)(1+v)}
$$




$$
\begin{aligned}
& D_{111122}=\frac{r^{2} E(7-13 v)}{35(1-2 v)(1+v)} \\
& D_{111212}=\frac{3 r^{2} E v}{7(1-2 v)(1+v)} \\
& D_{122122}=\frac{3 r^{2} E(7-23 v)}{35(1-2 v)(1+v)}
\end{aligned}
$$

where the following relations hold

$$
\begin{aligned}
& D_{111111}=D_{222222} \\
& D_{111122}=D_{112112}=D_{112121}=D_{121121}=D_{211222}=D_{212212}=D_{212221}=D_{221221} \\
& D_{111212}=D_{111221}=D_{112211}=D_{112222}=D_{121211}=D_{121222}=D_{122212}=D_{122221} \\
& D_{122122}=D_{211211}
\end{aligned}
$$

The other elements of $C_{i j k l}$ and $D_{i j g k l m}$ are all zero. Note that (1) Eqs.(23) and (24) provide a useful method for estimating the high-order constitutive constants directly from the Young's modulus and Poisson's ratio without explicitly knowing the numerical values of either the number of contacts $N$ or the representative volume $V$; and (2) the derived higher-order constitutive coefficients explicitly depend upon the particle radius, $r$, which functions as a internal length scale parameter.

In a damage context we assume that all constitutive coefficients are pre-multiplied with the same factor $(1-\omega)$ such that a nonlinear higher-order constitutive damage model can be obtained as:

$$
\begin{aligned}
\sigma_{i q}^{0} & =(1-\omega) C_{i q k l} \varepsilon_{k l}^{0} \\
\sigma_{i j q}^{1} & =(1-\omega) D_{i j q k l m} \varepsilon_{k l m}^{1}
\end{aligned}
$$

where $\omega$ is the so-called damage scalar quantity ranging from 0 for initial undamaged material to 1 when all material coherence is lost. For the calculations in this paper, the damage state is governed by a linear strain softening damage law through a scalar state variable, $k$, defined as the overall effective strain. The effective strain, $k$, is determined by the square root of the summation of principle strains considering damage due to only tensile strains, which, in $2 \mathrm{D}$, is given by the following equation

$$
k=\sqrt{\left(\varepsilon_{1}\right)^{2}+\left(\varepsilon_{2}\right)^{2}} \quad \text { for } \varepsilon_{1}, \varepsilon_{2}>0
$$

where $\varepsilon_{1}$ and $\varepsilon_{2}$ are the principle strain components of strain $\varepsilon=\left[\begin{array}{ll}\varepsilon_{11} & \varepsilon_{12} \\ \varepsilon_{12} & \varepsilon_{22}\end{array}\right]$. The linear softening damage evolution function takes the form 


$$
\omega(k)= \begin{cases}\frac{k_{u}\left(k-k_{0}\right)}{k\left(k_{u}-k_{0}\right)} & k_{0} \leq k \leq k_{u} \\ 1 & k>k_{u}\end{cases}
$$

where $k_{0}$ is the threshold of strain at which damage is initiated and $k_{u}$ is the strain level at which all load carrying capacity is exhausted.

\section{Energy functional and weak form}

Following the framework for strain-gradient theory (Germain 1973, Fleck and Hutchinson 1997), the strain potential energy density with higher-order stress can be expressed as

$$
W=\int_{\varepsilon^{0}} \sigma_{i q}^{0} d \varepsilon_{i q}^{0}+\int_{\varepsilon^{1}} \sigma_{i j q}^{1} d \varepsilon_{i j q}^{1}
$$

To proceed, we substitute the damage constitutive relations from Eqs. (27) and (28) into Eq. (31) and make use of integration by parts for the higher-order term while ignoring the boundary terms such that the final form of the energy functional can be recast as

$$
\begin{aligned}
W & =\int_{\varepsilon^{0}}(1-\omega) C_{i q k l} \varepsilon_{k l}^{0} d \varepsilon_{i q}^{0}-\int_{\varepsilon^{0}}(1-\omega) D_{i j q k l r} \frac{\partial^{2} \varepsilon_{k l}^{0}}{\partial x_{r} \partial x_{j}} d \varepsilon_{i q}^{0} \\
& +\int_{\varepsilon^{0}} \frac{\partial \omega}{\partial \varepsilon_{m n}^{0}} \frac{\partial \varepsilon_{m n}^{0}}{\partial x_{j}} D_{i j q k l r} \frac{\partial \varepsilon_{k l}^{0}}{\partial x_{r}} d \varepsilon_{i q}^{0}
\end{aligned}
$$

where the substitution $\varepsilon_{i j q}^{\mathrm{I}}=\partial \varepsilon_{i q}^{0} / \partial x_{j}$ has been used. Minimization of the potential energy results in the following nonlinear equilibrium equation in terms of displacement gradients:

$$
\begin{aligned}
& (1-\omega)\left[C_{i q k l} \frac{\partial^{2} u_{k}}{\partial x_{q} \partial x_{l}}-D_{i j q k l r} \frac{\partial^{4} u_{k}}{\partial x_{r} \partial x_{j} \partial x_{q} \partial x_{l}}\right] \\
& -\frac{\partial \omega}{\partial \varepsilon_{m n}^{0}} \frac{\partial^{2} u_{m}}{\partial x_{q} \partial x_{n}}\left[C_{i q k l} \frac{\partial u_{k}}{\partial x_{l}}-D_{i j q k l r} \frac{\partial^{3} u_{k}}{\partial x_{r} \partial x_{j} \partial x_{l}}\right] \\
& +\frac{\partial \omega}{\partial \varepsilon_{m n}^{0}} D_{i j q k l r}\left[\frac{\partial^{3} u_{m}}{\partial x_{j} \partial x_{q} \partial x_{n}} \frac{\partial^{2} u_{k}}{\partial x_{r} \partial x_{l}}+\frac{\partial^{2} u_{m}}{\partial x_{j} \partial x_{n}} \frac{\partial^{3} u_{k}}{\partial x_{r} \partial x_{q} \partial x_{l}}\right]=0
\end{aligned}
$$

Pre-multiplying Eq. (33) by a test function $\delta u_{i}$ and integrating over the $2 \mathrm{D}$ domain $\Omega$, the weak form governing equation is obtained as (see Yang and Misra 2010 for derivation details)

$$
\begin{aligned}
& \int_{\Omega} \frac{\partial \delta u_{i}}{\partial x_{q}}(1-\omega) C_{i q k l} \frac{\partial u_{k}}{\partial x_{l}} d x d y+\int_{\Omega} \frac{\partial^{2} \delta u_{i}}{\partial x_{q} \partial x_{j}}(1-\omega) D_{i j q k l r} \frac{\partial^{2} u_{k}}{\partial x_{r} \partial x_{l}} d x d y \\
& =\int_{\Gamma_{t}} \frac{\partial \delta u_{i}}{\partial x_{q}} \sigma_{i j q}^{1} n_{j} d \Gamma+\int_{\Gamma_{t}} \delta u_{i}\left(\sigma_{i q}^{0}-\frac{\partial \sigma_{i j q}^{1}}{\partial x_{j}}\right) n_{q} d \Gamma
\end{aligned}
$$

According to Reddy (2005), terms corresponding to the test function in the boundary integrals are determined as the essential boundary conditions, while their coefficients form the natural boundary conditions. Thus, the boundary conditions for this higher-order equilibrium system can be stated as 
Essential b.c.: $\quad u_{i}$ specified, $u_{i, q}$ specified

Natural b.c.: $\quad \sigma_{i q}^{0}-\frac{\partial \sigma_{i j q}^{1}}{\partial x_{j}}$ specified, $\sigma_{i j q}^{1}$ specified

Note that though boundary conditions have been identified, the physical significance of the natural boundary conditions resulting from higher-order stresses remains an open question.

\section{Enforcement of essential boundary conditions using penalty method}

The natural boundary conditions (or traction boundary conditions) have been included into the weak form equilibrium equation via integration by parts. However, the essential boundary conditions (or displacement boundary conditions) have not yet been treated in the formulation. Moreover, the Moving Least Square (MLS) approximations used latter in EFG discretization do not bear the Kronecker delta function property. Therefore, the essential boundary conditions have to be imposed separately via special techniques, such as the Lagrange multiplier method and the penalty method. Penalty method offers an efficient way to impose the essential boundary conditions provided an appropriate large penalty coefficient is utilized. The constrained higher-order Galerkin weak form (Eq. (34)) using penalty method is posed as follows (see also Liu and $\mathrm{Gu} 2005$ ):

$$
\begin{aligned}
& \int_{\Omega} \frac{\partial \delta u_{i}}{\partial x_{q}}(1-\omega) C_{i q k l} \frac{\partial u_{k}}{\partial x_{l}} d x d y+\int_{\Omega} \frac{\partial^{2} \delta u_{i}}{\partial x_{q} \partial x_{j}}(1-\omega) D_{i j q k l r} \frac{\partial^{2} u_{k}}{\partial x_{r} \partial x_{l}} d x d y \\
& -\int_{\Gamma_{t}} \frac{\partial \delta u_{i}}{\partial x_{q}} \sigma_{i j q}^{1} n_{j} d \Gamma-\int_{\Gamma_{t}} \delta u_{i}\left(\sigma_{i q}^{0}-\frac{\partial \sigma_{i j q}^{1}}{\partial x_{j}}\right) n_{q} d \Gamma \\
& -\frac{1}{2} \int_{\Gamma_{u}} \delta\left[\left(u_{i}-\bar{u}_{i}\right)^{T} \alpha\left(u_{i}-\bar{u}_{i}\right)\right] d \Gamma=0
\end{aligned}
$$

where $\bar{u}_{i}$ is the prescribed displacement vector; $\alpha$ is the penalty coefficient which is often a large positive number and is determined herein by $10^{6}$ times the maximum diagonal element of the global stiffness matrix. In Eq. (36), the higher-order essential boundary conditions are ignored for the sake of simplicity, though it could be included in an obvious and straightforward manner. Considering that

$$
\frac{1}{2} \int_{\Gamma_{u}} \delta\left[\left(u_{i}-\bar{u}_{i}\right)^{T} \alpha\left(u_{i}-\bar{u}_{i}\right)\right] d \Gamma=\int_{\Gamma_{u}} \delta u_{i}^{T} \alpha u_{i} d \Gamma-\int_{\Gamma_{u}} \delta u_{i}^{T} \alpha \bar{u}_{i} d \Gamma
$$

Eq.(36) can be recast as

$$
\begin{aligned}
& \int_{\Omega} \frac{\partial \delta u_{i}}{\partial x_{q}}(1-\omega) C_{i q k l} \frac{\partial u_{k}}{\partial x_{l}} d x d y+\int_{\Omega} \frac{\partial^{2} \delta u_{i}}{\partial x_{q} \partial x_{j}}(1-\omega) D_{i j q k l r} \frac{\partial^{2} u_{k}}{\partial x_{r} \partial x_{l}} d x d y \\
& -\int_{\Gamma_{u}} \delta u_{i}^{T} \alpha u_{i} d \Gamma=\int_{\Gamma_{t}} \frac{\partial \delta u_{i}}{\partial x_{q}} \sigma_{i j q}^{1} n_{j} d \Gamma+\int_{\Gamma_{t}} \delta u_{i}\left(\sigma_{i q}^{0}-\frac{\partial \sigma_{i j q}^{1}}{\partial x_{j}}\right) n_{q} d \Gamma \\
& -\int_{\Gamma_{u}} \delta u_{i}^{T} \alpha \bar{u}_{i} d \Gamma=0
\end{aligned}
$$




\section{Element-free Galerkin Formulation}

Meshfree methods, such as the Element-free Galerkin (EFG) methods, have been used as an alternative to eliminate the mesh-subjectivity. EFG method, which requires a much looser topological discretization structure compared to the finite element method, has been demonstrated to be quite successful in solving many challenging problems in solid mechanics, for instance, static and dynamic crack growth modeling (Krysl and Belytschko 1997; Belytschko and Tabbara 1996; Belytschko et al. 1994, 1995; Belytschko et al. 1995; Belytschko et al. 1994; Lu et al. 1994). However, there have been only few attempts to apply the EFG method to gradient-enhanced continua with strain softening (Askes et al. 2000; Chang et al. 2002) or in the context of plasticity (Pamin et al. 2001, 2003). Jirásek (1998) has investigated the applicability of EFG method to strain softening problems and confirmed that for regularized localization problems, EFG method behaves in a manner superior to finite element (FE) method in the description of continuous fields. From the viewpoint of gradient-enhanced continuum theory developed in this paper, the EFG method has an important advantage over classical FE method that the approximation functions with high order of continuity needed for proper representation of the higher-order derivatives can be readily incorporated into the formulation without increasing the problem size (Askes et al. 2000; Pamin et al. 2003).

The essential idea for the EFG method is that MLS interpolants are used for the trail and test functions with a variational principle. To use MLS, it is only necessary to construct a set of nodes in the problem domain without any elements. The connectivity between field nodes is satisfied via the overlapping of the domain of influence of sampling node in which its shape function is nonzero. The domain of influence of each field node is controlled by a weight function. The weight and MLS shape functions used in this work are given in Appendix A. Using the MLS approximation, the trial function and test function are discretized according to:

$u_{i}=\phi_{i p} \underline{u}_{p} \quad \delta u_{i}=\phi_{i p} \delta \underline{u}_{p}$

where $\phi_{i p}$ is the MLS shape function and $\underline{u}_{p}$ is the nodal parameter of displacement field for all nodes in the influence domain. Substituting Eq. (39) into the weak form Eq. (38) and canceling out $\delta \underline{u}_{p}$ because of its arbitrariness yields the following global discretized system equation

$\left[K_{p s}+K_{p s}^{\alpha}\right] \underline{u}_{s}=F_{p}+F_{p}^{\alpha}$

where superscript $\alpha$ represents the resultants from penalty terms. Global stiffness tensors $K_{p s}, K_{p s}^{\alpha}$ and global force tensors $F_{p}, F_{p}^{\alpha}$ are given as

$K_{p s}=\int_{\Omega} \frac{\partial \phi_{i p}^{T}}{\partial x_{q}}(1-\omega) C_{i q k l} \frac{\partial \phi_{k s}}{\partial x_{l}} d x d y+\int_{\Omega} \frac{\partial^{2} \phi_{i p}^{T}}{\partial x_{q} \partial x_{j}}(1-\omega) D_{i j q k k r} \frac{\partial^{2} \phi_{k s}}{\partial x_{r} \partial x_{l}} d x d y$ 
$K_{p s}^{\alpha}=-\int_{\Gamma_{u}} \phi_{i p}^{T} \alpha \phi_{i s} d \Gamma$

$F_{p}=\int_{\Gamma_{t}} \frac{\partial \phi_{i p}^{T}}{\partial x_{q}} \sigma_{i j q}^{1} n_{j} d \Gamma+\int_{\Gamma_{t}} \phi_{i p}^{T}\left(\sigma_{i q}^{0}-\frac{\partial \sigma_{i j q}^{1}}{\partial x_{j}}\right) n_{q} d \Gamma$

$F_{p}^{\alpha}=-\int_{\Gamma_{u}} \phi_{i p}^{T} \alpha \bar{u}_{i} d \Gamma$

In order to obtain the incremental form of system Eq. (40), we define a residual force $R_{p}$ as the difference between internal force $\left(K_{p s}+K_{p s}^{\alpha}\right) \underline{u}_{s}$ and external force $F_{p}+F_{p}^{\alpha}$. Taylor series expansion of the residual force is then utilized to perform the linearization given by

$R_{p}=R_{p}\left(u^{(r-1)}\right)+\left(\frac{\partial R_{p}}{\partial \underline{u}_{s}}\right)^{(r-1)} \Delta \underline{u}_{s}^{(r)}+\frac{1}{2}\left(\frac{\partial^{2} R_{p}}{\partial \underline{u}_{s}^{2}}\right)^{(r-1)}\left(\Delta \underline{u}_{s}^{(r)}\right)^{2} \cdots=0$

where $\Delta \underline{u}^{(r)}$ is the increment and superscripts within parentheses refer to the iteration step. Thus the solution of Eq. (40) at the $r$ th iteration can be written in terms of the solution for the (r-1)th iteration as follows

$u^{(r)}=u^{(r-1)}+\Delta u^{(r)}$

When second-order derivatives and higher in Eq. (45) are neglected, we obtain

$$
\left(\frac{\partial R_{p}}{\partial \underline{u}_{s}}\right)^{(r-1)} \Delta \underline{u}_{s}^{(r)}=F_{p}+F_{p}^{\alpha}-\left(K_{p s}^{(r-1)}+K_{p s}^{\alpha(r-1)}\right) \underline{u}_{s}^{(r-1)}
$$

Defining tangent stiffness as $T_{p s}=\frac{\partial R_{p}}{\partial \underline{u}_{s}}$ yields

$T_{p s}=\sum_{m=1}^{n} \frac{\partial\left(K_{p m}+K_{p m}^{\alpha}\right)}{\partial \underline{u}_{s}} \underline{u}_{m}+\left(K_{p s}+K_{p s}^{\alpha}\right)$

Inserting Eq. (41) and Eq. (42) into Eq. (48), the resultant tangent stiffness tensor is obtained as

$$
\begin{aligned}
& T_{p s}=\int_{\Omega} \frac{\partial \phi_{i p}^{T}}{\partial x_{q}}(1-\omega) C_{i q k l} \frac{\partial \phi_{k s}}{\partial x_{l}} d x d y+\int_{\Omega} \frac{\partial^{2} \phi_{i p}^{T}}{\partial x_{q} \partial x_{j}}(1-\omega) D_{i j q k l r} \frac{\partial^{2} \phi_{k s}}{\partial x_{r} \partial x_{l}} d x d y \\
& -\int_{\Gamma_{u}} \phi_{i p}^{T} \alpha \phi_{i s} d \Gamma-\int_{\Omega} \frac{\partial \phi_{i p}^{T}}{\partial x_{q}} \frac{\partial \omega}{\partial \varepsilon_{a b}^{0}} \frac{\partial \phi_{a s}}{\partial x_{b}} C_{i q k l} \varepsilon_{k l}^{0} d x d y \\
& -\int_{\Omega} \frac{\partial^{2} \phi_{i p}^{T}}{\partial x_{q} \partial x_{j}} \frac{\partial \omega}{\partial \varepsilon_{a b}^{0}} \frac{\partial \phi_{a s}}{\partial x_{b}} D_{i j q k l r} \frac{\partial \varepsilon_{k l}^{0}}{\partial x_{r}} d x d y
\end{aligned}
$$

Finally, the incremental system equilibrium equation becomes 
$T_{p s}^{(r-1)} \Delta \underline{u}_{s}^{(r)}=F_{p}+F_{p}^{\alpha}-\left(K_{p s}^{(r-1)}+K_{p s}^{\alpha(r-1)}\right) \underline{u}_{s}^{(r-1)}$

\section{IGF Failure - Comparison of $\boldsymbol{A b}$ initio and Continuum Simulations}

We apply the derived theory to simulate failure of an IGF model in which a glassy film is embedded between two crystallites.

\section{Atomic model and ab initio failure simulation}

A fully relaxed 907-atom initial periodic super-cell atomic model of nanometer sized IGF sandwiched between $\beta-\mathrm{Si}_{3} \mathrm{~N}_{4}$ crystals was generated and, subsequently subjected to uniaxial extension. The details of model construction and the tensile simulation are given in Ching et al. (2010). Here we provide information relevant for comparison with continuum modeling and needed for completeness. The initial IGF model was constructed using classical molecular dynamics followed by full ab initio relaxation using the VASP (Vienna ab-initio Simulation Package). VASP is a popular electronic structure code based on density functional theory (Kresse and Furthmuller 1996a, b, Hafner 2008) with plane wave expansion within the pseudopotential formulism. We used Vanderbilt ultrasoft pseudopotential with LDA for exchange-correlation potential and a relatively high energy cutoff of 500 $\mathrm{eV}$ (Ching et al. 2010). A criterion of $10^{-5} \mathrm{eV}$ for electronic convergence and $10^{-2} \mathrm{eV} / \mathrm{A}$ for force convergence were adopted. The stress level for the equilibrium structure is below $0.1 \mathrm{GPa}$. The initial model has a dimension of $14.533 \AA$ x $15.225 \AA$ x $47.420 \AA$ and an IGF width of approximately $1.64 \mathrm{~nm}$. From this initial model, uniaxial extension was applied in small incremental steps by stretching the supercell model. The $\mathrm{y}$ - and z-dimensions of the model were kept constant while the $\mathrm{x}$-dimension is increased such that the strain components were $\varepsilon_{x x} \neq 0$, while $\varepsilon_{y y}=\varepsilon_{z z}=0$. At each strain-level (referred to the entire periodic model), all atoms in the model were fully relaxed using the same criteria as in the initial model until the desired convergence is achieved. During ab initio simulations under uniaxial loading, small changes in atomic positions can result in large unbalanced forces. Therefore, it is necessary to apply stringent convergence criterion. The convergence in the total energy and the atomic strain level were carefully monitored to ensure sufficient accuracy without consuming a prohibitive amount of computing resources. The relaxed model at a given strain serves as the starting position for the next increment of strain before the model is fully relaxed again. Depending on the stress level, relaxation run could take several hundred ionic steps to reach the desired convergence. This process is carried on until the total energy and the stress data show that the "sample" is fully fractured or reached the deformation limit. The data for the atomic structure of the IGF model are collected as a function of strain for further analysis.

\section{Continuum model and failure simulation with higher order theory}

For assessing the ability of the higher-order continuum approach in replicating the behavior predicted by $a b$ initio simulations, we constructed a 2D IGF model cell of dimension $50 \AA \times 15 \AA$. Since $a b$ initio simulations were under a uniaxial extension, 2D model was considered sufficient. The model was subjected to uniaxial tension via an incrementally imposed displacement $d u=0.2 \AA$ at the right end 
while the left end are fixed at both $\mathrm{x}$ and $\mathrm{y}$ direction and the upper and lower boundaries are fixed at the y-direction as shown in Figure 2. For simplicity, a $10 \AA$ wide imperfection zone, shown as the hatched area in Figure 2, is considered along the center section of the domain to represent the IGF. The elastic modulus of the crystal region is taken as $E=350 \mathrm{GPa}$ (Ching et al. 2010). The elastic modulus of the IGF layer is specified to be 56GPa. The IGF modulus is obtained from the mixture rules of compliances based upon the average slope of the $a b$ initio stress-strain curve estimated as $170 \mathrm{GPa}$ and the crystal modulus given above. Poisson's ratio was taken as $v=0.22$, and the damage evolution parameters are given as $k_{0}=0.18$ and $k_{u}=0.6$ for glassy film; $k_{0}=0.2$ and $k_{u}=0.6$ for crystal. A $51 \times 11$ uniform nodal layout is used for obtaining the solutions for this problem. In addition, $40 \times 12$ rectangular background cells with four-point integration rule are used to integrate the stiffness coefficient. Since the unit cell of $\beta-\mathrm{Si}_{3} \mathrm{~N}_{4}$ has a tetrahedral structure; we chose the side length of the tetrahedra as the representative length scale parameter $r$. Based upon a Si-N bond length of $1.7 \AA$, the length scale parameter $r$ was specified as $2.94 \AA$. The incremental displacement was imposed in twenty two stages until the IGF model completely fractured.

\section{Results and Discussion}

Figure 3 gives the comparison of the stress-strain curves obtained from ab initio simulations and the continuum modeling. The agreement between the curves obtained from the two methods is encouraging considering that the continuum model is based upon (1) a linear elastic law with a linear softening damage law, and (2) an idealized IGF geometry with homogeneous properties.

Figure 4 gives the ball-stick view at selected strain-levels of how the atomic positions and bonding evolve under the uniaxial extension-loading. We define the atomic bonds between Si-N and $\mathrm{Si}-\mathrm{O}$ upon the basis of atomic distances for the purposes of depicting the evolution of bonding in the ball-stick view. In crystals, the $\mathrm{Si}-\mathrm{O}$ and $\mathrm{Si}-\mathrm{N}$ bond lengths are $1.61 \AA$ in $\alpha-\mathrm{SiO}_{2}$ and $1.66-1.70 \AA$ in various oxynitride crystals, respectively (Ching 2004, Ching et al. 2004). In our ball-stick diagrams, the bond is deemed broken when the atomic distances exceed the sum of the covalent radii. In Figure 4, the strain-level of 0.069 represents a point in the pre-peak on-linear portion of the stress-strain curve, the strain-level of 0.088 is the peak stress point and the strain-level of 0.108 is a point past peak stress in the softening regime of the stress-strain curve. At the strain-level of 0.069 , the ball-stick diagram shows minimal change in the structure. Upon further extension, at the strain-level of 0.088 , a considerable number of bonds at the IGF/crystal interface are broken, although the atomic structure shows a seemingly small change both in the crystal layers and within the IGF. At the strain-level of 0.108 , the IGF experiences large stretch while the adjoining crystal layers rebound or unload. At this stage, void spaces and "nano-fractures" appear within the IGF. In comparison, laboratory tests on silicon nitride under uniaxial stress loading show a failure strain of $\sim 2.5 \%$ (Edwards et al. 2004). We note here that the supercell considered in this study is not a representative of the lab sample. In addition, the failure of a laboratory sample is through a more complex process under multi-axial local strains and highly defective structures, including larger scale defects. 
To compare the local deformation behavior obtained from the two methods, we computed the local strain field of the atomic system by fitting the atomic displacements obtained from the ab initio simulations. The super-cell was divided into a 51x11x11 grid. A linear fit was defined for local groups of atoms contained within a sphere of radius $R_{o}$ centered at the $\mathrm{n}^{\text {th }}$ grid point, such that the displacement of $\mathrm{p}^{\text {th }}$ atom within this group, is given as:

$u_{i}^{n p}=a_{i}^{n}+d_{i j}^{n} X_{j}^{p}$

where, $u_{i}^{n p}$ is the fitted displacement for the $\mathrm{p}^{\text {th }}$ atom, $X_{j}^{p}$ is the position of the $\mathrm{p}^{\text {th }}$ atom in the unstressed configuration, the coefficients $a_{i}^{n}$ denote the rigid body displacement, and the coefficients $d_{i j}{ }^{n}$ represent the local displacement gradient. Local strain associated with the $\mathrm{n}^{\text {th }}$ grid point was taken as the symmetric part of the displacement gradient. In our analysis, $R_{o}$ is chosen to be $5.0 \AA$ based upon two considerations: (1) to ensure a minimal local volume that guarantees the existence of solutions to the fitting process used to obtain the local displacement gradient; and (2) to obtain a maximum resolution for the strain field in the IGF region as a too large a $R_{o}$ will average out the local variations of the strain field. The strain components were averaged over the z-direction for comparing with the $2 \mathrm{D}$ continuum simulations.

Figures 5 through 7 show the computed contours of strain $\varepsilon_{11}$ in the horizontal direction $(x$ direction), strain $\varepsilon_{22}$ in the vertical direction ( $y$ direction) and shear strain $\varepsilon_{12}$ at three stages, namely, pre-peak, peak and post-peak failure stages from the continuum and $a b$ initio simulations, respectively. The three stages for the continuum simulation correspond to the overall strains of (a) 0.067 , (b) 0.071 and (c) 0.086, while that for atomistic simulation correspond to the values in Figure 4. The agreements between the continuum and ab initio simulation results are encouraging. We see from Figure 5 that the continuum result replicates the horizontal direction strain, $\varepsilon_{11}$, localization in the IGF and the post-peak rebound of the crystal region. We further see from Figure 6 that vertical strain, $\varepsilon_{22}$, also concentrate in the IGF and form a pattern of alternate compressive and tensile strain band. Similarly, from Figure 7 we observe that shear strains form a cross pattern in the vicinity of the IGF. The strain localization zone in the continuum model is observed to be narrower than the $a b$ initio simulation, since the lower estimate of imperfection width was chosen. Wider localization zone would be obtained if the initial imperfection width was assumed to be larger. We also note that the continuum model directly provides the predictions of the local strain while for the ab initio results the local strain has to be interpreted through a fitting process.

Figure 8 further illustrates the evolution of damage and strain localization predicted by the continuum model by plotting the damage function, $\omega$, and axial strain, $\varepsilon_{11}$, along the horizontal central axis for all the loading steps. We observe that as the damage initiates, a localized strain zone begins to emerge within the IGF. This localized zone grows till we reach the peak stress. Beyond peak stress the localization zone is confined to an unchanging narrow band and the crystal region experiences unloading as shown in Fig. 8(b).

Figure 9 plots the contours of the higher-order strains $\varepsilon_{111}$ and $\varepsilon_{222}$ corresponding to the gradients 
of the horizontal strain $\varepsilon_{11}$ and vertical strain $\varepsilon_{22}$ at the peak and post-peak failure stages. As the strains localize within the IGF, strain gradients develop in their proximity. At peak axial stress stage, two strain gradient bands with mirror symmetry form on the either side of the imperfection as shown in Figure 9(a). At failure, shown in Figure 9(b), bands of large strain gradients are present in the immediate neighborhood of the rupture while the rest of the material experiences zero strain gradients which are consistent with the strain profiles shown in Figures 5-6.

\section{Summary and Conclusion}

This paper has presented a micro-structural granular mechanics based higher order stress-strain theory for fracture simulation of strain softening materials. In this approach, the constitutive coefficients are derived by considering the underlying physical configuration such that the internal length scale parameter reflects the natural granularity of the underlying microstructure. The resultant higher order theory includes both strain gradients and their conjugate higher-order stress, which is different from other gradient theories. The constitutive relationships, the governing equations and its weak form have been derived for this higher-order theory in this paper. An Element-free Galerkin (EFG) formulation in combination with the penalty method for the enforcement of essential boundary conditions is then applied for the discretization of the system governing equations followed by the linearization using Talyor series expansions. The derived formulation is used to simulate the fracture process of glassy inter-granular films (IGF) sandwiched between crystal layers. The results predicted by the higher order continuum model are compared to those obtained from $a b$ initio atomistic simulations. The similarity between the continuum and ab initio simulation results are encouraging considering that the continuum model (1) is based upon a linear elastic law with a linear softening damage law, (2) uses an idealized IGF geometry with homogeneous properties, and (3) directly provides the predictions of the local strain while for the $a b$ initio results the local strain has to be interpreted through a fitting process. In addition, the internal length parameter utilized in this approach not only serves as a localization limiter but also rationally reflects the micro-structural characteristics of the IGF model which enables this higher-order continuum theory to simulate the fracture process of nano-scale complex materials accurately from numerical viewpoint without losing their physical significance. We also note that even with the currently available unprecedented computational resources ab initio solutions cannot be obtained routinely for such complex structures with $\sim 1000$ atoms let alone for macro-scale mechanical behavior of nano-phased materials that are suffused with IGF type structures. The micro-structural granular mechanics based higher order continuum approach developed in this paper offers a viable method that can greatly reduce the computational needs and provide realistic simulations. In our future studies, we will extend the 2D continuum simulations to $3 \mathrm{D}$, and apply anisotropic micro-structure to further refine this higher-order continuum theory.

\section{Acknowledgement:}

A.M. was partially supported by a NASA EPSCOR Partnership Development Grant. W.Y.C. is supported by the U.S. Department of Energy, Office of Basic Energy Sciences, Division of Materials Science and Engineering under the Grant No. DE-FG02-84DR45170. This research used the resources of 
NERSC supported by the Office of Science of DOE under the contract No. DE-AC03-76SF00098.

\section{References:}

Altan, B. S. and Aifantis, E. C. (1997). "On some aspects in the special theory of gradient elasticity." Journal of the Mechanical Behavior of Materials, 8, 231-282.

Askes, H., Pamin, J., de Borst, R. (2000). "Dispersion analysis and element-free Galerkin solutions of second- and fourth-order gradient enhanced damage models." International Journal of Numerical Methods in Engineering, 49, 811-832.

Bažant, Z. P. (1976). "Instability, ductility, and size effect in strain-softening concrete." Journal of the Engineering Mechanics Division, 102(2), 331-344.

Bažant, Z. P., Belytschko, T. and Chang, T. P. (1984). "Continuum theory for strain-softening." Journal of Engineering Mechanics, 110(2), 666-1692.

Bažant, Z.P. and Pijaudier-Cabot, G. (1988). "Nonlocal continuum damage, localization instability and convergence." Journal of Applied Mechanics, 55, 287-293.

Belytschko, T. B., Bažant, Z. P., Hyun, Y. W., Chang, T. P. (1986). "Strain-softening materials and finite-element solutions." Computers and Structures, 23(2), 163-180.

Belytschko, T., Gu, L. and Lu, Y. Y. (1994). "Fracture and crack growth by element free Galerkin methods." Modeling and Simulation in Materials Science and Engineering, 2( 3A), 519-534.

Belytschko, T., Lu, Y. Y.and Gu,. L. (1994). "Element-free Galerkin methods.” International Journal for Numerical Methods in Engineering, 37(2), 229-256.

Belytschko, T., Lu, Y. Y. and Gu, L. (1995). "Crack propagation by element-free Galerkin methods." Engineering Fracture Mechanics, 51(2), 295-315.

Belytschko, T., Lu, Y. Y. and Gu, L., Tabbara, M. (1995). "Element-free Galerkin methods for static and dynamic fracture.” International Journal of Solids and Structures, 32(17-18), 2547-2570.

Belytschko, T. and Tabbara, M. (1996). "Dynamic fracture using element-free Galerkin methods." International Journal for Numerical Methods in Engineering, 39, 923-938.

Chang, C. S., Askes, H., Sluys, L. J. (2002). "Higher-order strain/higher-order stress gradient models derived from a discrete microstructure, with application to fracture." Engineering Fracture Mechianics, 69, 1907-1924.

Chang, C. S. and Gao, J. (1995). "Second-gradient constitutive theory for granular material with random packing structure.” International Journal of Solids and Structures, 32(16), 2279-2293.

Chang, C. S. and Gao, J. (1997). "Wave propagation in granular rod using high-gradient theory." Journal of Engineering Mechanics, 123, 52-59.

Chang, C. S., Gao, J. and Zhong, X. (1998). "High-gradient modeling for love wave propagation in geological materials.” ASCE: Journal of Engineering Mechanics, 124, 1354-1359.

Chang, C. S. and Liao, C. L. (1990). "Constitutive relation for a particulate medium with the effect of particle rotation." International Journal of Solids and Structures, 26(4), 437-453.

Chang, C. S., Ma, L. (1990). "Modeling of discrete granulates as micropolar continua." Journal of Engineering Mechanics, 116(12), 2703-2721.

Chang, C. S. and Misra, A. (1990). "Packing structure and mechanical properties of granulates." Journal of Engineering Mechanics, 116(5), 1077-1093. 
Chen J, Ouyang L, Rulis P, Misra A, Ching WY. (2005). "Complex nonlinear deformation of nanometer intergranular glassy films in $\beta-\mathrm{Si}_{3} \mathrm{~N}_{4}$." Physical Review Letters, 95(25), 256103.

Chen, J. S., Wu, C. T., Belytschko, T. (2000). "Regularization of material instabilities by meshfree approximations with intrinsic length scales." International Journal for Numerical Methods in Engineering, 47, 1303-1322.

Ching, W.Y. (2004). "Electronic structure and bonding of all crystalline phases in the $\mathrm{SiO}_{2}-\mathrm{Y}_{2} \mathrm{O}_{3}-\mathrm{Si}_{3} \mathrm{~N}_{4}$ phase equilibrium diagram." Journal of the American Ceramic Society, 87, 1996-2013.

Ching, W.Y., Ouyang, L., Yao. H., Xu. Y.N. (2004). "Electronic structure and bonding in the Y-Si-O-N quaternary crystals.” Physical Review B, 70, 085105.

Ching W.Y., Rulis P, Ouyang L, Misra A. (2009). "Ab initio tensile experiment on a model of intergranular glassy film in $\beta-\mathrm{Si}_{3} \mathrm{~N}_{4}$ with prismatic surfaces." Applied Physics Letters, 94, 051907.

Ching, W.Y., Rulis, P., Ouyang, L., Misra, A. (2010). "A theoretical study of the elasticity, mechanical behavior, electronic structure, interatomic bonding, and dielectric function of an intergranular glassy film model in prismatic $\beta-\mathrm{Si}_{3} \mathrm{~N}_{4}$." Physical Review $B, 81,214120$.

de Borst, R. and Mühlhaus, H. B. (1992). "Gradient-dependent plasticity: Formulation and algorithmic aspects.” International Journal for Numerical Methods in Engineering, 35, 21-39.

de Borst, R., Pamin, J., Peerlings, R. H. J. and Sluys, L. J. (1995). "On gradient-enhanced damage and plasticity models for failure in quasi-brittle and frictional materials." Computational Mechanics, 17(1-2), 130-142.

de Borst, R., Sluys, L. J., Mühlhaus, H. B., Pamin, J. (1993). "Fundamental issues in finite element analyses of localization of deformation." Engineering Computations, 10, 99-121.

de Vree, J. H. P., Brekelmans, W. A. M. and van Gils, M. A. J. (1995). "Comparison of nonlocal approaches in continuum damage mechanics." Computers and Structures, 55, 581-588.

Edwards, R.L., Coles, G., Sharpe, W.N. (2004). "Comparison of tensile and bulge tests for thin-film silicon nitride." Experimental Mechanics, 44, 49-54.

Fleck, N. A. and Hutchinson, J. W. (1997). "Strain gradient plasticity." Advances in Applied Mechanics, 33, 295-361.

Frantziskonis, G. and Desai, C. S. (1987). "Analysis of a strain softening constitutive model." International Journal of Solids and Structures, 23(6), 751-767.

Germain, P. (1973) “The method of virtual power in continuum mechanics. Part 2: Microstructure." SIAM Journal of Applied Mathematics, 25(3), 556-575

Hafner, J. (2008). "Ab-initio simulation of materials using VASP: Density functional theory and beyond." Journal of Computational Chemistry, 29, 2044-2078.

Huang, H. and Van Swygenhoven H. (2009). "Atomistic simulations of mechanics of nanostructures." MRS Bulletin, 34, 160-162.

Jirásek, M. (1998). "Element-free Galerkin method applied to strain-softening materials." Proceedings of EURO-C, International Conference Computational Modelling of Concrete Structures, Balkema, Rotterdam/Brookfield, 1, A.A, 311-319.

Koch, C., Ovid'ko I., Seal, S., Veprek, S.(2007). "Structural Nanocrystalline Materials: Fundamentals and Applications." Cambridge University Press, Cambridge, U.K..

Kresse, G., Furthmuller, J. (1996a). "Efficiency of ab initio total energy calculation for metals and 
Semiconductors using plane wave basis set." Computational Materials Science., 6(1),15-50.

Kresse, G., Furthmüller, J. (1996b). "Efficient iterative schemes for ab initio total-energy calculations using a plane-wave basis set.” Physical Review B, 54(16), 11169-11186.

Krysl P. and Belytschko, T. (1997). "Propagation of 3D cracks by the element free Galerkin method." Fourth U.S. National Congress on Computational Mechanics, San Francisco.

Liu, G. R.and Gu, Y. T. (2005). "An Introduction to Meshfree Methods and Their Programming." Springer., ordrecht, New York.

Lu, Y. Y., Belytschko, T. and Tabbara, M. (1994). "Element-free Galerkin methods for wave propagation and dynamic fracture." Computer methods in Applied Mechanics and Engineering, 126(1-2), 131-153.

Luo J. (2007). "Stabilization of nanoscale quasi-liquid interfacial films in inorganic materials: A review and critical assessment." Critical Reviews in Solid State and Materials Science , 32(1-2), 67-109.

Mindlin, R.D. (1969). "Microstructure in linear elasticity." Archive for Rational Mechanics and Analysis, 16(1), 51-78.

Misra, A., Ouyang, L., Chen, J., and Ching, W.Y. (2007). "Ab initio calculations of strain fields and failure patterns in silicon nitride intergranular glassy films.” Philosophical Magazine, 87(25), 3839-3852.

Misra, A. and Yang, Y., (2010) "Micromechanical model for cohesive materials based upon pseudo-granular structure," International Journal of Solids and Structures, 47(21), 2970-2981.

Mühlhaus, H.B. and Oka, F. (1996). "Dispersion and wave propagation in discrete and continuous models for granular materials." International Journal of Solids and Structures, 33, 2841-2858.

Murakam, H., Kendall, D.M. and Valanis, K.C. (1993). "A nonlocal elastic damage theory: Mesh-Insensitivity under strain softening." Computers and Structures, 48(3), 415-422.

Needleman, A. (1988). "Material rate dependence and mesh sensitivity in localization problems." Computer Methods in Applied Mechanics and Engineering, 67, 69-85.

Nemes, J.A. and Spéciel, E. (1996). "Use of a rate-dependent continuum damage model to describe strain-softening in laminated composites.” Computers and Structures, 58(6), 1083-1092.

Ovid'ko, I.A. (2007). "Review on the fracture processes in nanocrystalline materials." Journal of Materials Science, 42(5), 1694-1708.

Pamin, J. (1994). "Gradient-dependent plasticity in numerical simulation of localization phenomena." doctoral dissertation, Delft University of Technology, The Netherlands.

Pamin, J., Askes, H., de Borst, R. (2001). "Gradient regularization and EFG discretization of the plastic flow theory." in: K.-U. Bletzinger, K. Schweizerhof, W.A. Wall (Eds.), Conference on Trends in Computational Structural Mechanics, CIMNE, Barcelona, 179-188.

Pamin, J., Askes, H., de Borst, R. (2003). "Two gradient plasticity theories discretized with the element-free Galerkin method." Computer Methods in Applied Mechanics and Engineering, 192, 2377-2403.

Pan, X. (1996). “Atomistic Structure of Silicon Nitride/Silicate Glass Interfaces.” Journal of the American Ceramic Society, 79(11), 2975-2979.

Peerlings, R.H.J., de Borst, R., Brekelmans, W.A.M., de Vree, J.H.P. (1996). “Gradient enhanced damage for quasi-brittle materials." International Journal for Numerical Methods in Engineering, 39(19), 3391-3403.

Phillips, R. (2001). “Crystal, Defects and Microstructures.” Cambridge University Press, Cambridge, 
U.K.

Pietruszczak, St. and Mroz, Z. (1981). "Finite element analysis of deformation of strain-softening materials." International Journal for Numerical Methods in Engineering, 17, 327-334.

Reddy, J.N. (2005). "An introduction to finite element method ( ${ }^{\text {rd }}$ Edition)." McGraw-Hill Science/Engineering/Math.

Sandler, I.S. (1984). "Strain softening for static and dynamic problems." ASME Winter Annual Meeting, Symp. On constitutive equations: Micro, Macro and Computational Aspects, CEQ, New Orleans, 217-231.

Shibata, N., Pennycook, S., Gosnell, T., Painter, G., Shelton, W., and Becher, P. (2004). "Observations of rare-earth segregation in silicon nitride ceramics at subnanometer dimensions." Nature, 428, 730.

Sluys, L.J. (1992). "Wave propagation, localization and dispersion in softening solids." Doctoral dissertation, Delft University of Technology, The Netherlands.

Sluys, L. J. and de Borst, R. (1992). "Wave-propagation and localization in a rate-dependent cracked medium model formulation and one-dimensional examples." International Journal of Solids and Structures, 29. 2945-2958.

Sluys, L. J., de Borst, R. and Mühlhaus, H. B. (1993). "Wave propagation, localization and dispersion in a gradient-dependent medium.” International Journal of Solids and Structures, 30, 1153-1171.

Steinmann, P. (1994). “An improved FE expansion for micropolar localization analysis." Communications in Numerical Methods in Engineering. 10(12), 1005-1012.

Subramaniam, A., Koch, C.T., Cannon, R.M. and Rühle, M.(2006). "Intergranular glassy films: An overview." Materials Science and Engineering, 422, 3.

Suiker, A.S.J., de Borst, R. and Chang, C.S. (2001a). "Micro-mechanical modeling of granular material, part 1-Derivation of a second-gradient micro-polar constitutive theory." Acta Mechanica, 149(1-4), 161-180.

Suiker, A. S. J., de Borst, R. and Chang, C. S. (2001b). "Micro-mechanical modeling of granular material. part 2-Plane wave propagation in infinite media." Acta Mechanica, 149(1-4), 181-200.

Sutton, A.P. and Balluffi, R.W. (1995). "Interfaces in Crystalline Materials." Clarendon Press, Oxford, U.K.

Triantafyllidis, N. and Bardenhagen, S. (1993). "On higher order gradient continuum theories in 1-D nonlinear elasticity. Derivation from and comparison to the corresponding discrete models." Journal of Elasticity, 33, 259-293.

Valanis, K.C. (1991). “A global damage theory and the hyperbolicity of the wave problem." ASME Journal of applied Mechanics, 58(2), 311-316.

$\mathrm{Wu}$, F.H. and Freund, L.B. (1984). "Deformation trapping due to thermoplastic instability in one dimensional wave propagation." Journal of Mechanics and Physics of Solids, 32(2), 119-132.

Yang, Y. and Misra, A., (2010) "Higher-order stress-strain theory for damage modeling implemented in an element-free Galerkin formulation." Computer Modeling in Engineering and Sciences, 64(1), 1-36.

Zhu, T., Li, J., Ogata, S., Yip, S. (2009). "Mechanics of ultra-strength materials.” MRS Bulletin, 34, 167. Ziegler, A., Idrobo, J., Cinibulk, M., Kisielowski, C., Browning, N., Ritchie, R. (2004). "Interface structure and atomic bonding characteristics in silicon nitride ceramics." Science, 306, 1768. 


\section{Appendix A. MLS approximation}

For a 2D case considered in this work, the weight function $w_{i}(x)$ is obtained as the product of standard 1D weight functions in $x$ and $y$ directions given as

$$
w_{i}(x)=w_{i x}(x) \cdot w_{i y}(x)
$$

In this study, a cubic spline is used as the weight function and the domain of influence is set to be rectangular with dimension $d_{s x}$ and $d_{s y}$ which are determined by a dimensionless parameter $\beta$ and the nodal spacing $d_{c x}$ and $d_{c y}$ in each direction respectively. For instance, the weight function in $x$ direction takes the following form

$$
w_{i x}(x)=\left\{\begin{array}{lr}
2 / 3-4 \bar{r}_{i x}^{2}+4 \bar{r}_{i x}^{3} & \bar{r}_{i x} \leq 0.5 \\
4 / 3-4 \bar{r}_{i x}+4 \bar{r}_{i x}^{2}-4 / 3 \bar{r}_{i x}^{3} & 0.5<\bar{r}_{i x} \leq 1 \\
0 & \bar{r}_{i x} \geq 1
\end{array}\right.
$$

where $\bar{r}_{i x}=\frac{\left|x-x_{i}\right|}{d_{s x}}, d_{s x}=\beta d_{c x}($ with $\beta=3)$ and $\left|x-x_{i}\right|$ is the distance from node $x_{i}$ to the sampling point $x$.

The MLS approximation $u^{h}(x)$ for displacement field function $u(x)$ at $x$ is defined as

$$
u^{h}(x)=\Phi^{T}(x) U_{s}
$$

where $U_{s}$ is the vector collecting the nodal parameters of displacement field for all the nodes within the influence domain; $\Phi^{T}(x)$ is the vector of MLS shape functions corresponding to $n$ nodes in the influence domain of the sampling point $x$, written as

$$
\Phi^{T}(x)=\left\{\phi_{1}(x) \phi_{2}(x) \cdots \phi_{n}(x)\right\}_{1 \times n}=p^{T}(x) A^{-1}(x) B(x)
$$

where the polynomial base vector $p$ takes the quadratic form as

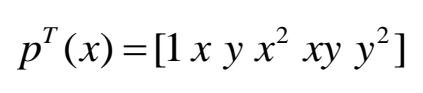

and the matrix $A(x)$ and vector $B(x)$ are given as

$$
\begin{aligned}
& A(x)=\sum_{i=1}^{n} w_{i}(x) p\left(x_{i}\right) p^{T}\left(x_{i}\right) \\
& B(x)=\left[w_{1}(x) p\left(x_{1}\right) w_{2}(x) p\left(x_{2}\right) \ldots w_{n}(x) p\left(x_{n}\right)\right]
\end{aligned}
$$




\section{List of Figures:}

Figure 1: Conceptual granular model of a continuum.

Figure 2: Prismatic IGF model and loading conditions.

Figure 3: Comparison of stress-strain curves with ab initio calculation results.

Figure 4: Ball-stick diagram of prismatic IGF model deformation obtained from ab initio calculations under x-direction uniaxial extension at three different loading-levels: (a) pre-peak, under strain of 0.069 (b) peak stage, under strain of 0.088 (c) post-peak failure stage, under strain of 0.108 , respectively.

Figure 5: Evolution of axial strain $\varepsilon_{l 1}$ contours at three stages: (a) pre-peak, (b) peak, and (c) post-peak failure stages.

Figure 6: Evolution of lateral strain $\varepsilon_{22}$ contours at three stages: (a) pre-peak, (b) peak, and (c) post-peak failure stages.

Figure 7: Evolution of shear strain $\varepsilon_{12}$ contours at three stages: (a) pre-peak, (b) peak, and (c) post-peak failure stages.

Figure 8: Evolution of (a) damage $\omega$ and (b) axial strain $\varepsilon_{1 l}$ along the horizontal central axis over all the loading steps $(51 \times 11$ nodes, $v=0.22, r=2.94 \AA)$.

Figure 9: Contours of strain gradients $\varepsilon_{111}$ and $\varepsilon_{222}$ at: (a) peak, and (b) post-peak failure stages. 


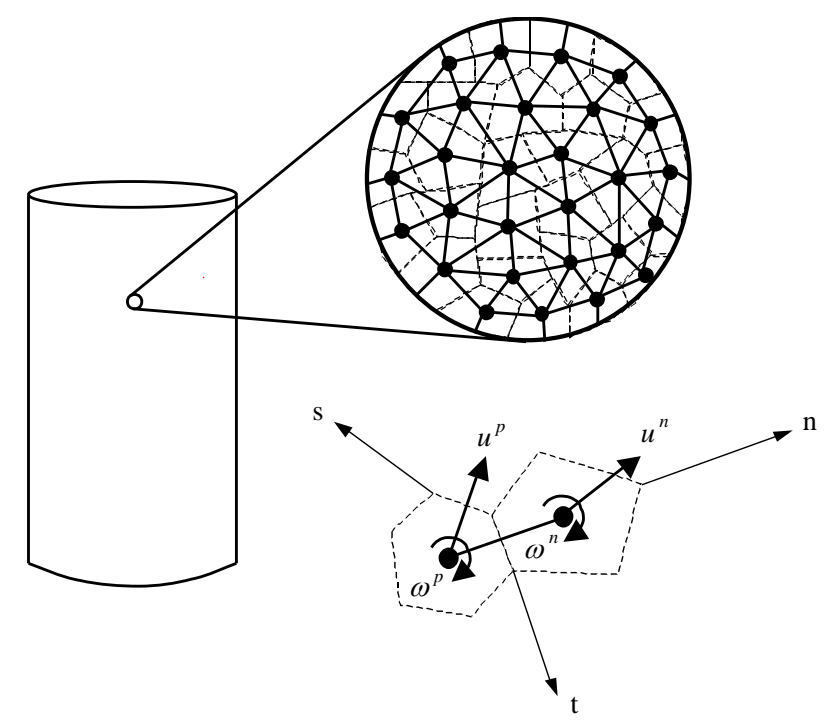

Figure 1: Conceptual granular model of a continuum 


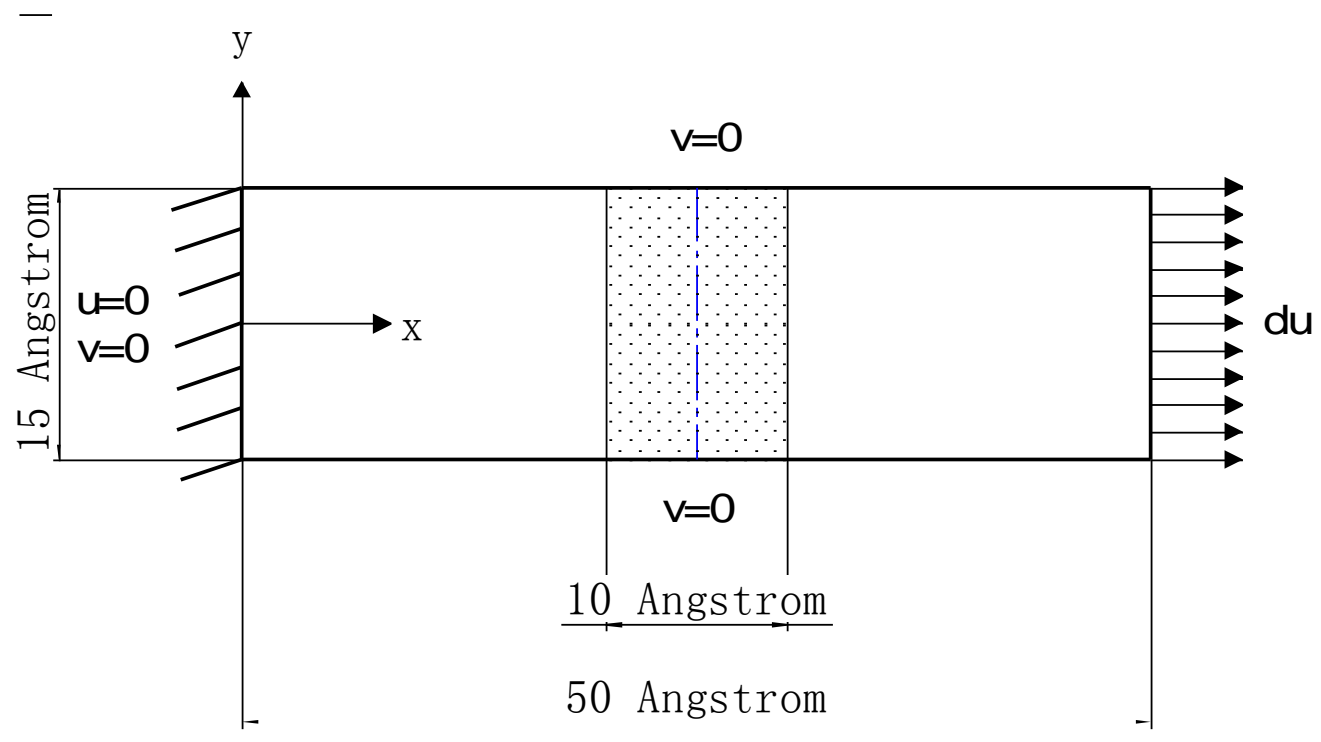

Figure 2: Prismatic IGF model and loading conditions 


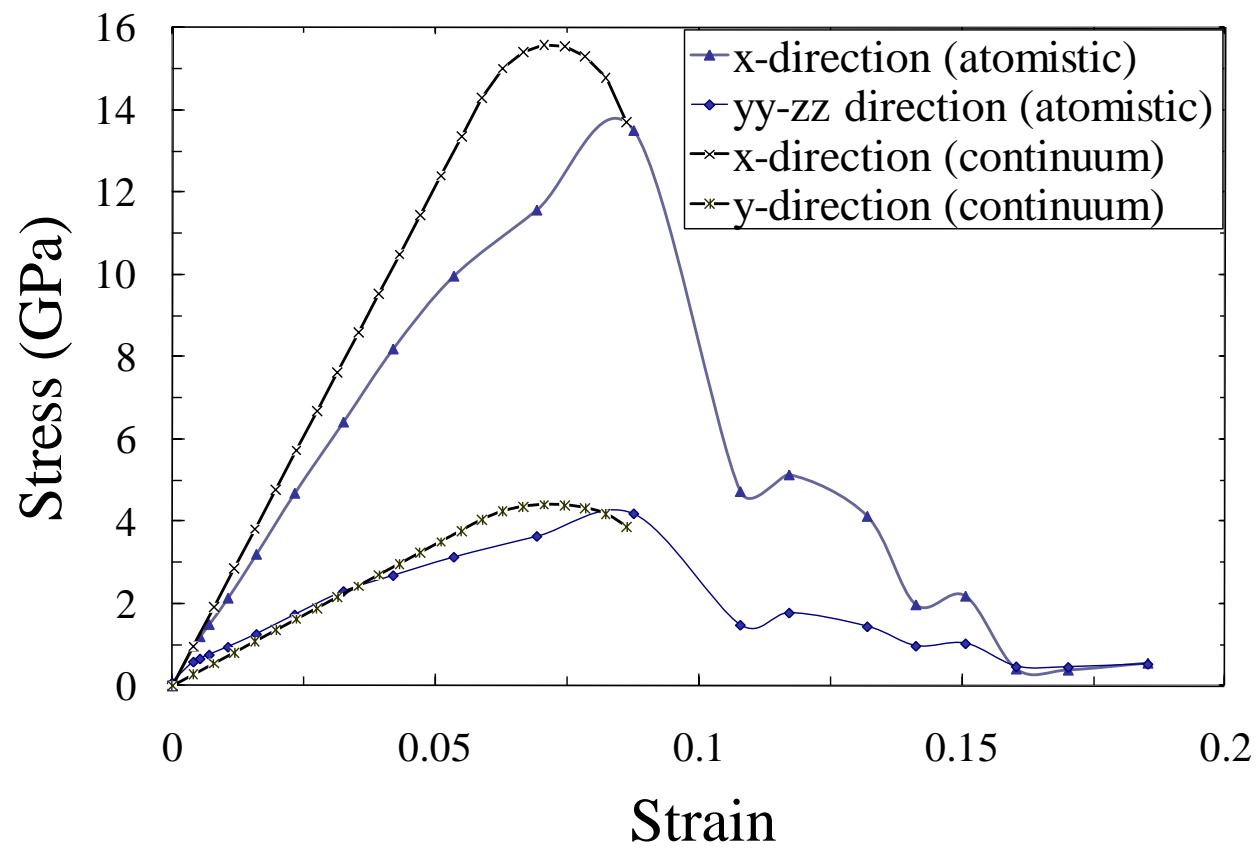

Figure 3: Comparison of stress-strain curves with $a b$ initio calculation results 
(a)

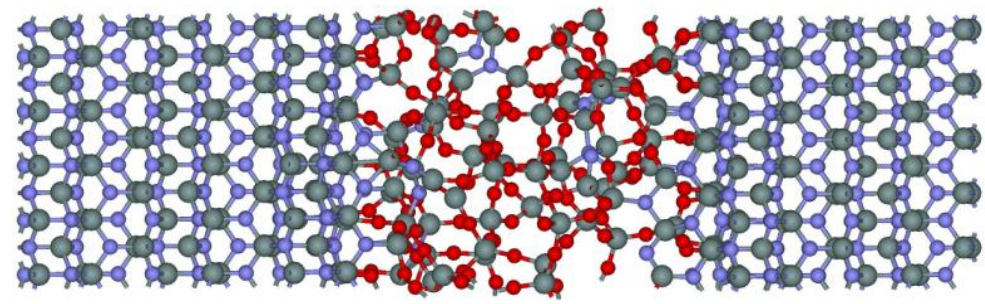

(b)

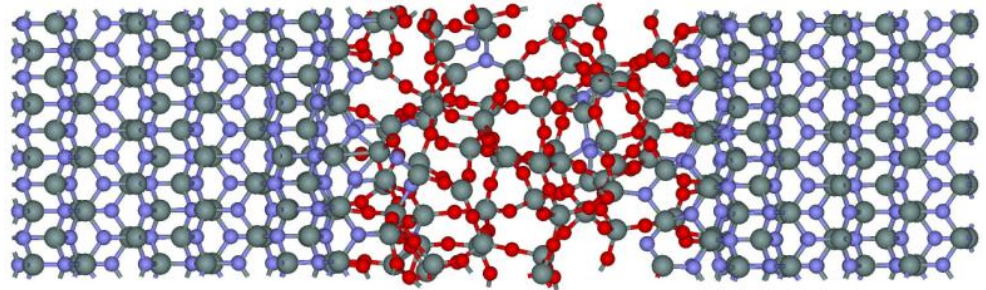

(c)

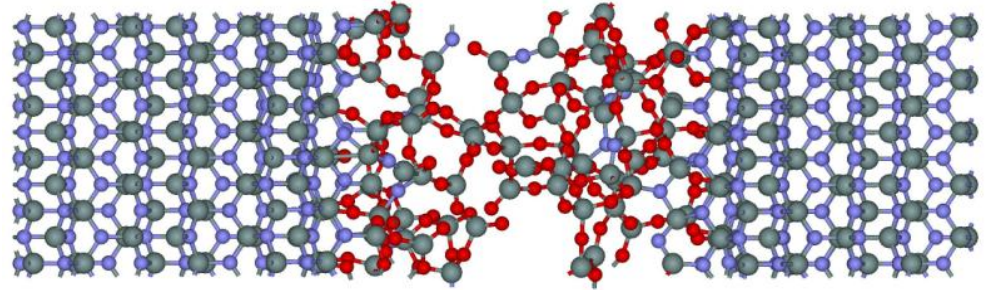

Silicon

- Nitrogen

- Oxygen

Figure 4: Ball-stick diagram of prismatic IGF model deformation obtained from ab initio calculations under X-direction uniaxial extension at three different loading-levels: (a) pre-peak, under strain of 0.069 (b) peak stage, under strain of 0.088 (c) post-peak failure stage, under strain of 0.108 , respectively 


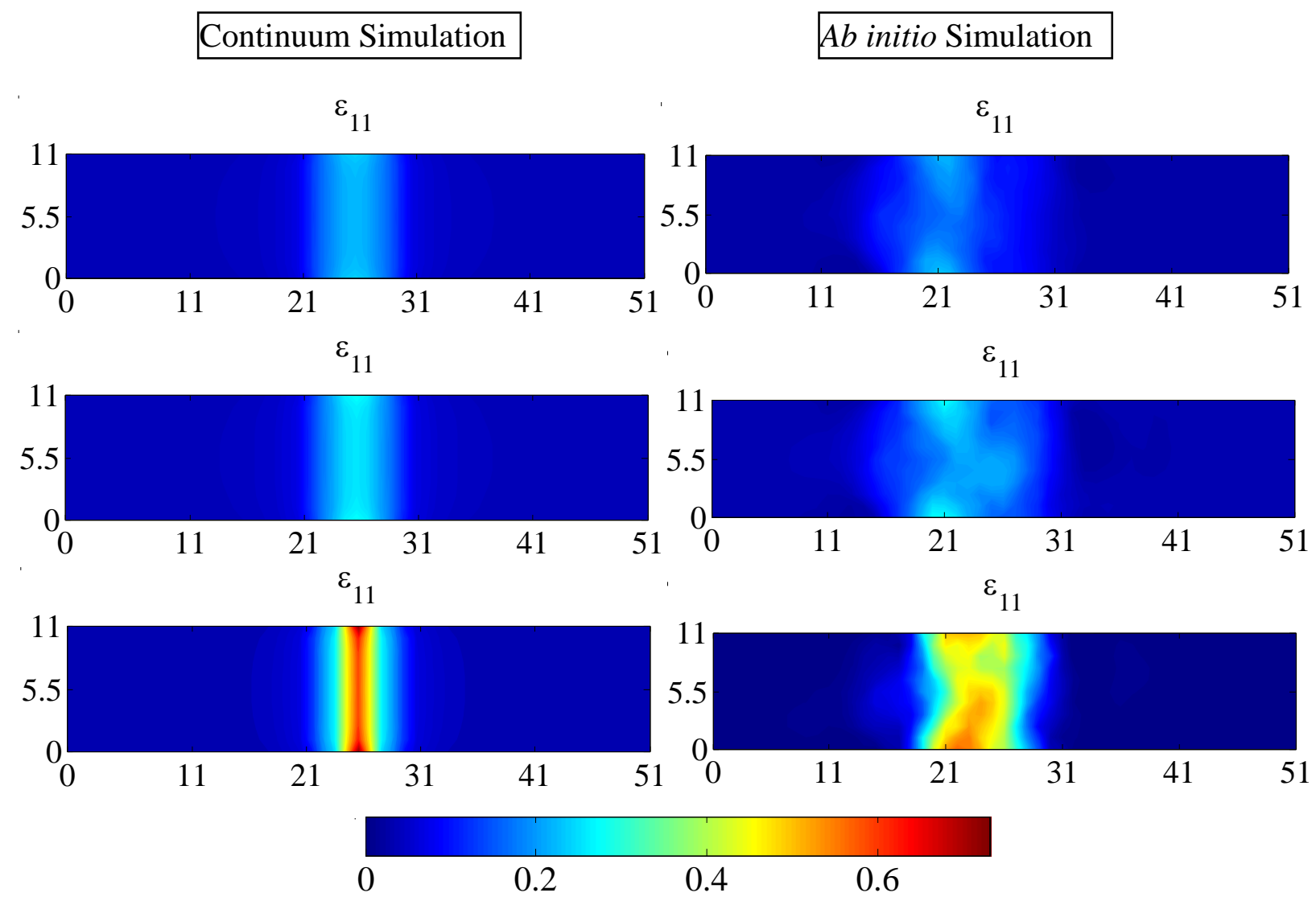

Figure 5: Evolution of axial strain $\varepsilon_{l l}$ contours at three stages: (a) pre-peak, (b) peak, and (c) post-peak failure stages 


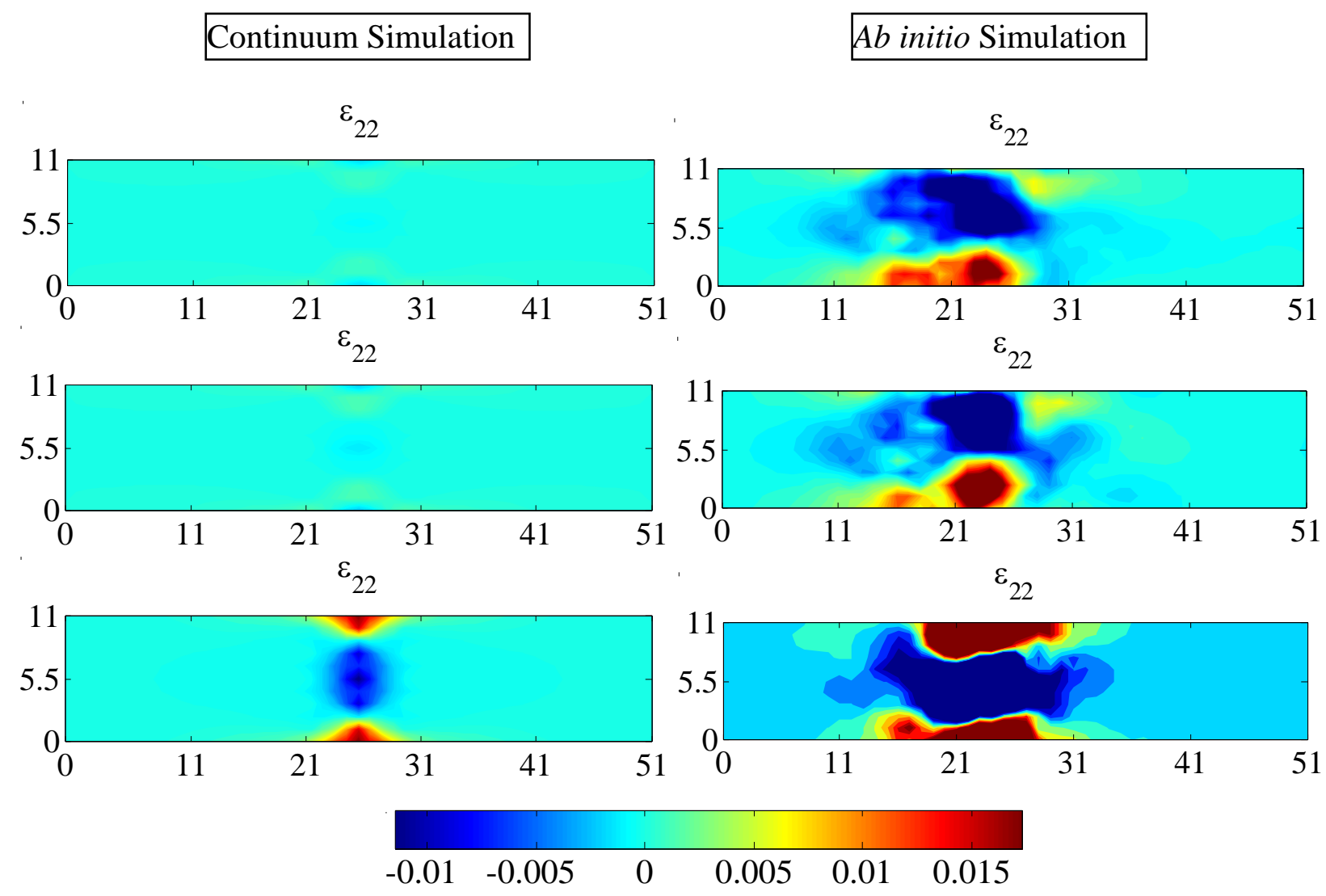

Figure 6: Evolution of lateral strain $\varepsilon_{22}$ contours at three stages: (a) pre-peak, (b) peak, and (c) post-peak failure stages 
Continuum Simulation

$\varepsilon_{12}$
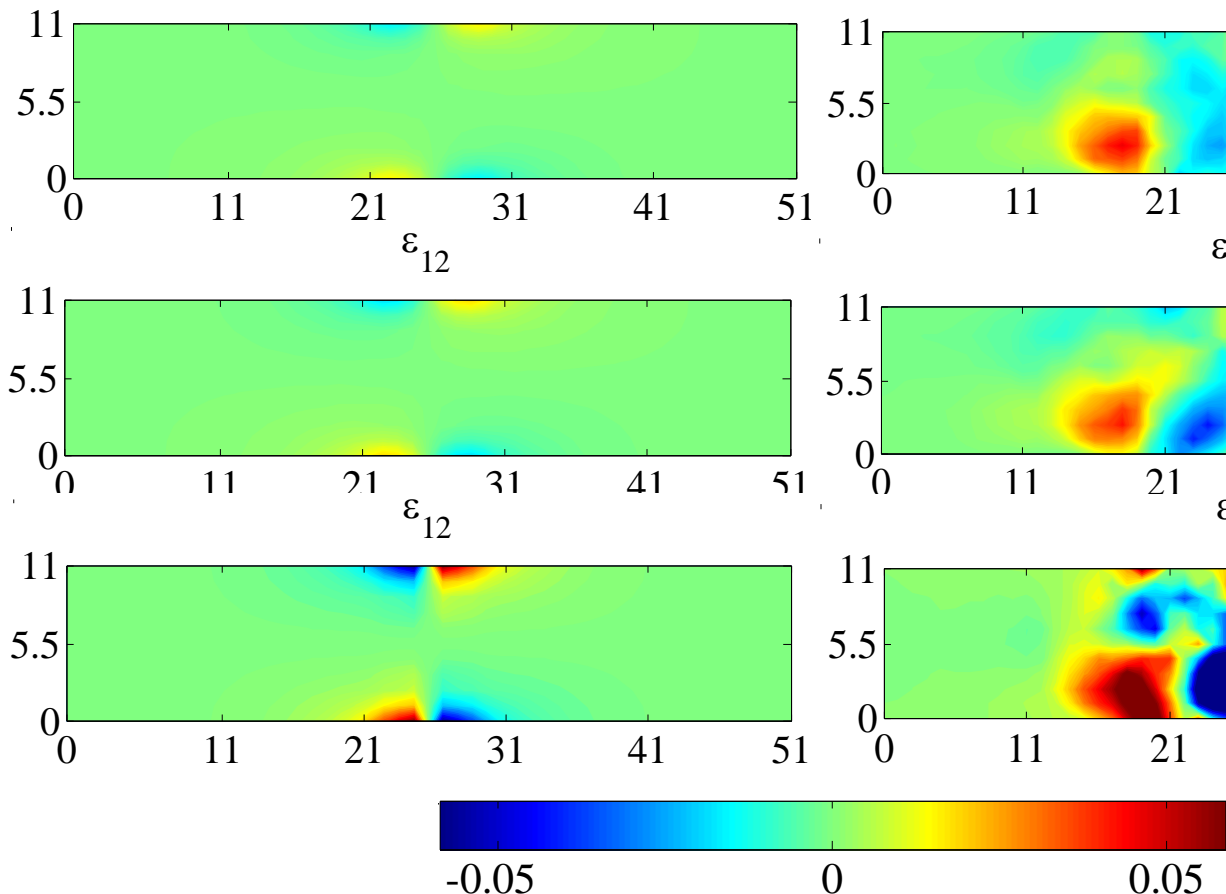

Figure 7: Evolution of shear strain $\varepsilon_{12}$ contours at three stages: (a) pre-peak, (b) peak, and (c) post-peak failure stages
Ab initio Simulation

$\varepsilon_{12}$
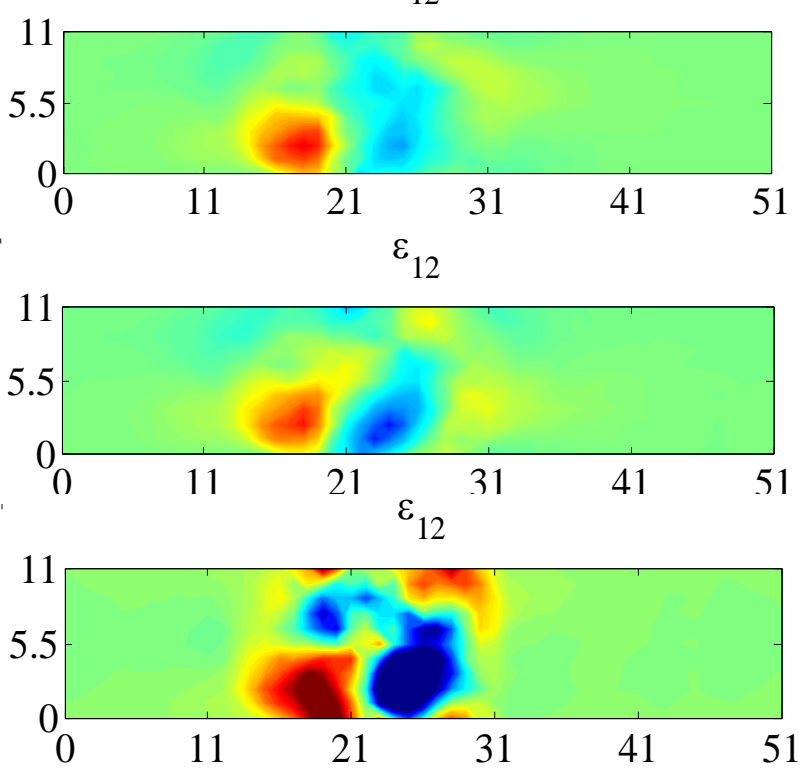

0.05 

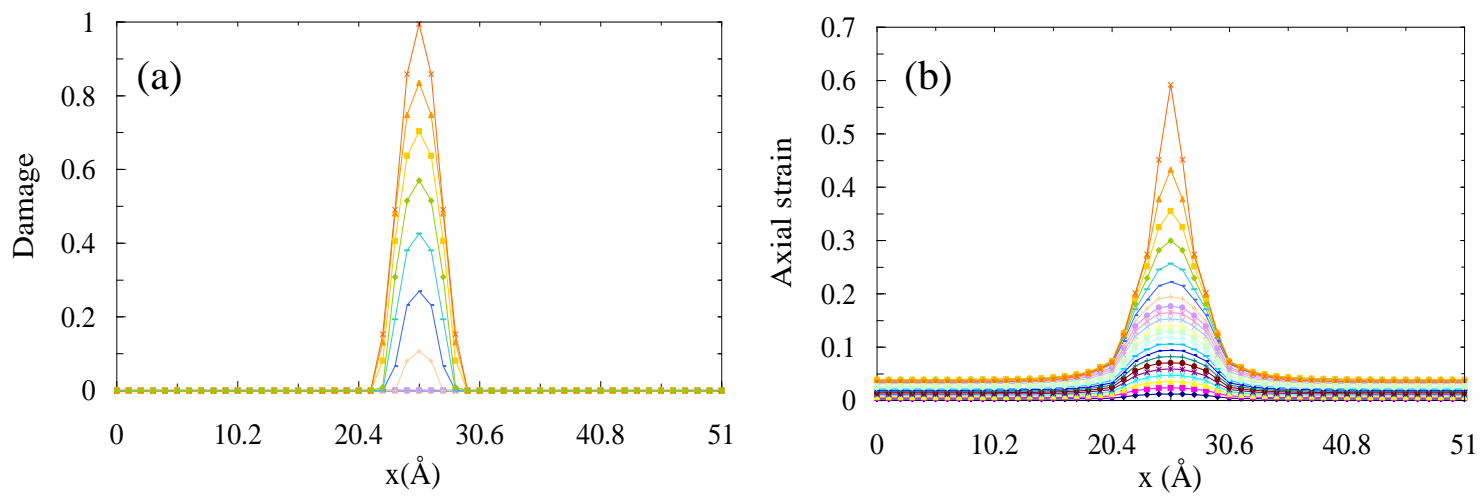

Figure 8: Evolution of (a) damage $\omega$ and (b) axial strain $\varepsilon_{11}$ along the horizontal central axis over all the loading steps $(51 \times 11$ nodes, $v=0.22, r=2.94 \AA)$ 

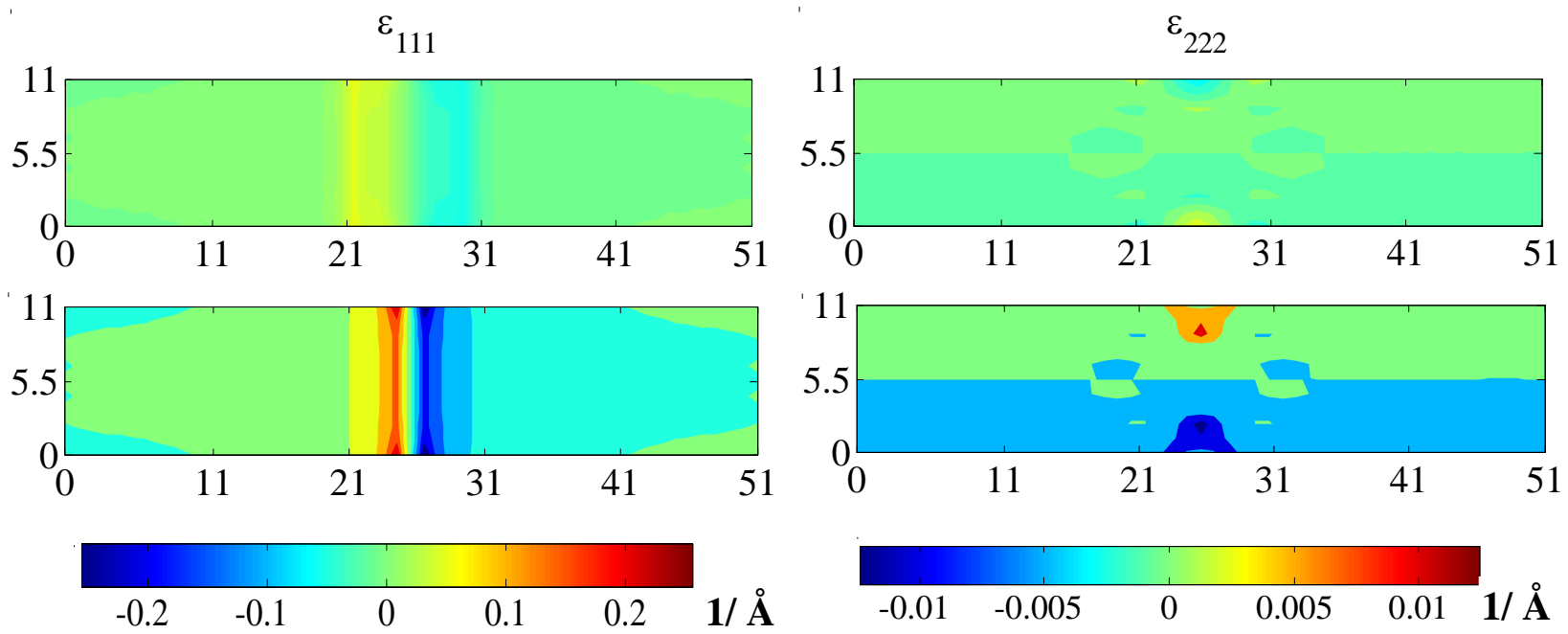

Figure 9: Contours of strain gradients $\varepsilon_{111}$ and $\varepsilon_{222}$ at: (a) peak, and (b) post-peak failure stages 